\title{
PREVENCIÓN DE LA OBESIDAD EN ESCUELAS BÁSICAS DE PEÑALOLÉN: COMPONENTE ALIMENTACIÓN Y NUTRICIÓN
}

\section{OBESITY PREVENTION IN PRIMARY SCHOOLS IN PEÑALOLEN: FOOD AND NUTRITION COMPONENT}

\author{
Fernando Vio del R. (1), Isabel Zacarías H. (1), Lydia Lera (1) \\ María Cecilia Benavides (1) Ana María Gutiérrez (2)
}

(1) Instituto de Nutrición y Tecnología de los Alimentos, INTA, Universidad de Chile. Santiago, Chile.

(2) Corporación Municipal de Peñalolén. Santiago, Chile.

\begin{abstract}
A food and nutrition education intervention was implemented in nine public schools in Peñalolen, Chile, with one control school. The objective was to determine the trend in obesity prevalence and in food knowledge and food consumption in preschool and school-age children followed longitudinally in the first year of intervention, compared with the control school. The sample included 1556 pre-school, first and second grade (1225 children in experimental schools and 331 in the control school) followed during one school year. Nutrition education activities were performed with teachers and students, and the evaluation of nutritional status was carried out in two points, March-April and October-November 2010. There was also one measurement of snack consumption in pre-school children and food and nutrition survey in school age children at the beginning and at the end of the year. Results showed that there was no change in overweight and obesity in the intervened school-age children, and a small increase in the control school. However, analyzing nutritional status by school, in seven of the nine experimental schools there was a decrease in obesity. A high percentage of preschool children carried soft drinks and juices to school. In school age children there was a significant increase in fruit and dairy consumption during the year. School age children had a good knowledge of fruits and dairy food, but not of fish and legumes. Despite the short period of food and nutrition intervention, this study shows that interventions implemented in a structured way with an evaluation can have a positive impact on changing eating habits and improving the nutritional status of municipal preschool and school children.
\end{abstract}

Key words: nutritional status evaluation, pre-school and school children obesity, food knowledge, food consumption.

Este trabajo fue recibido el 20 de Abril de 2011 y aceptado para ser publicado el 12 de Junio de 2011.

\section{INTRODUCCIÓN}

El incremento de la obesidad infantil en Chile ha llegado a ser un importante problema de salud pública y nutrición (1), el cual en vez de disminuir, aumenta año tras año, de acuerdo a la información de JUNAEB de la obesidad de los niños de primer año básico en escuelas municipales y particulares subvencionadas del país, hasta llegar a un $21 \%$ el año 2009 (2).

Ante esta situación, se han desarrollado diversas estrategias se han desarrollado, en especial intervenciones de alimentación y nutrición y actividad física derivadas del exitoso proyecto realizado en Casablanca entre los años 2002 y 2004, el cual demostró que al término del segundo año de intervención en las tres escuelas municipales de la comuna, la obesidad disminuyó en un $50 \%$ (3 - 5). A raíz de esta experiencia, se han replicado intervenciones en escuelas de todo el país a través del programa EGO escuelas del Ministerio de Salud (6) y del programa NutriRSE, de Acción Responsabilidad Social Empresarial (RSE) (7) en las comunas de Macul, Puente Alto, Maipú, Santiago Centro y Estación Central de la Región Metropolitana, financiado por empresas privadas desde el año 2006. Sin embargo, una evaluación del programa de las 1000 EGO escuelas no demostró avance en términos de reducción de la obesidad, la que ha seguido incrementándose, ni en los cambios al interior 
de las escuelas para mejorar la alimentación de los niños y aumentar su actividad física (8).

En el año 2010, considerando las experiencias anteriores, se inició un proyecto asociativo entre la Municipalidad de Peñalolén y la empresa Nestlé Chile, asesorados por el INTA, llamado "Niños en Acción". Este proyecto tiene como propósito fomentar estilos de vida saludable para mejorar el estado nutricional en la comunidad escolar de nueve colegios municipalizados de Peñalolén, con uno como control, con actividades en alimentación y nutrición y actividad física en alumnos de pre-kínder, kínder, primero y segundo año básico, por un periodo de 3 años. Durante el primer año, se elaboró una línea de base del estado nutricional al inicio del proyecto y se realizó una medición al término del primer año de intervención.

El objetivo de este trabajo fue evaluar los cambios en el estado nutricional, conocimiento y consumo de alimentos de los niños de pre-kínder, kínder, primero y segundo básico en nueve colegios al término del primer año de intervención, compararlos con el colegio control y analizar los resultados en el contexto de los planes y programas de educación nutricional que se están realizando en el país.

\section{MATERIAL Y MÉTODOS Diseño}

Se trata de un estudio cuasi-experimental, de se- guimiento longitudinal por un período de un año, en escolares de educación prebásica y básica de la comuna de Peñalolén.

\section{Sujetos}

El universo de prekinder, kínder, primero y segundo básico de los colegios seleccionados al inicio del estudio (línea base) y seguidos durante el año 2010, fue de 1556 niños. De estos, 1225 corresponden a los niños de las escuelas intervenidas y 331 a la escuela control, con una distribución semejante en niños y niñas, que está descrita en la tabla 1.

\section{Programa de Intervención}

El trabajo en terreno estuvo a cargo de dos nutricionistas responsables de la gestión y ejecución de actividades dentro de los establecimientos educacionales. Se capacitó al 95\% de los profesores al inicio de la intervención. La intervención educativa consistió en trabajar con 6 ejes temáticos basados en los mensajes de las guías alimentarias (9), pasando cada mes un tema diferente. Se realizó una sesión educativa mensual por curso, efectuada por nutricionista en aula, y se desarrollaron actividades referentes al tema durante el mes tales como concursos o visitas. Por ejemplo, en mayo se trabajó el consumo de pescado; en junio-julio el consumo de legumbres; en agosto lácteos, en septiembre frutas y verduras, y así sucesivamente. Para el diseño de las

\section{TABLA 1}

Distribución de la muestra por curso y género.

$\begin{array}{cccc}\text { Curso } & \text { Niños } & \text { Niñas } & \text { Total } \\ & n=758(48.7) & n=798(51.3) & n=1556\end{array}$

\section{Intervenidos}

Pre Kinder

104 (17.1)

117 (19.0)

219 (17.9)

Kínder

158 (26.0)

$142(23.0)$

$300(24.5)$

Primero básico

174 (28.4)

179 (29.0)

353 (28.7)

Segundo básico

174 (28.6)

$179(29.0)$

$353(28.8)$

Total intervenidos

608 (49.6)

$617(50.4)$

1225

\section{Control}

$\begin{array}{lr}\text { Pre Kinder } & 48(32.0) \\ \text { Kínder } & 40(26.7) \\ \text { Primero básico } & 30(20.0) \\ \text { Segundo básico } & 32(21.3) \\ \text { Total control } & 150(45.3)\end{array}$

47 (26.0)

95 (28.7)

48 (26.5)

88 (26.6)

49 (27.1)

79 (23.9)

$37(20.4)$

69 (20.8)

Total control

$181(54.7)$ 
actividades realizadas en aula se revisaron materiales educativos ya existentes y probados en intervenciones anteriores, tales como “i¿Qué rico es comer sano!!” (10) y otros elaborados por el INTA y por la Corporación 5 al día Chile sobre frutas y verduras (11). A partir de estos materiales, se generaron actividades educativas propias destinadas a los alumnos de cada curso (prekinder, kinder, primero y segundo básico), las que se centraron en aprender jugando.

Respecto a los kioskos al interior de los colegios, durante el año de intervención se capacitó a los encargados de cada uno de ellos en alimentación y kiosco saludable. La corporación municipal asumió un rol importante en el tema, modificando los contratos y estableciendo las bases de licitación para el año siguiente.

En el caso de la actividad física, la comuna de Peñalolén ya había instalado un programa que aumentaba de dos a cuatro las horas de educación física, separadas al menos en dos veces a la semana en todos los colegios municipales, con profesores de educación física y equipamiento para el desarrollo de las clases, lo cual funcionaba en todos los colegios al inicio del estudio. Por lo tanto, en este aspecto no hubo diferencias entre los nueve colegios intervenidos y el colegio control.

Este proyecto fue aprobado por el Comité de Ética del INTA, Universidad de Chile.

\section{Mediciones antropométricas}

Las mediciones antropométricas realizadas fueron de peso y talla. La recolección de información se efectuó en los meses de abril-mayo y noviembre-diciembre 2010, y fue responsabilidad de una de las nutricionistas del proyecto, siendo realizada por otras nutricionistas de la comuna debidamente capacitadas y supervisadas.

La información se recolectó en cada una de las escuelas y para ello se utilizaron balanzas Health o meter (balance de brazo basculante) de $100 \mathrm{~g}$ de precisión y $200 \mathrm{~kg}$ de capacidad y la talla se midió con estadiómetros portátiles marca SECA modelo 206 de $1 \mathrm{~mm}$ de precisión y 2,2 metros de capacidad y se consideraron los protocolos recomendados para antropometría de la OMS (12). Los datos fueron registrados en planillas especiales diseñadas para ello y los datos fueron procesados y analizados en el INTA.

\section{Medición del conocimiento y consumo de alimentos}

Con el propósito de conocer el tipo de alimentos que llevan como colación los niños de prekinder y kinder ( $\mathrm{n}=418$ de prekinder y $\mathrm{n}=546$ de kinder), se efectuó un registro en terreno mediante observación directa en un sólo día. La recolección de la información estuvo a cargo de dos nutricionistas. Para evaluar el consumo y conocimiento en los alumnos de primero y segundo básico (123 de primero y 152 de segundo), se utilizó una encuesta especialmente diseñada para este propósito, incluyendo un diseño grafico con dibujos de alimentos de fácil comprensión. El test tenía un total de once preguntas, de las cuales seis preguntas eran de conocimiento y cinco de consumo; en este último aspecto, se evaluó el consumo diario de verduras, frutas y lácteos, y consumo semanal de legumbres. El traspaso de la información recolectada, la validación de la misma y el análisis e interpretación de los resultados fue realizado por el grupo de profesionales del INTA.

\section{Análisis estadístico}

Se realizó un análisis descriptivo de las variables en el total de la muestra según género, intervención y curso (promedio \pm desviación estándar o porcentajes según el tipo de variable). Se calculó el IMC ( $\mathrm{kg} / \mathrm{m} 2)$ de cada niño con los datos de peso y talla. Se calcularon los puntajes z de IMC usando Anthro 2005 para los menores de 5 años (13) y OMS 2007 para el resto) (14). Se utilizó el puntaje $\mathrm{z}$ de IMC, considerando que el promedio del puntaje $\mathrm{z}$ representa una tendencia central del número de desviaciones estándar (DE) en que se encuentra la población estudiada, respecto de la mediana de la población de referencia $(13,14)$. Se determinó el estado nutricional (bajo peso, normal, sobrepeso y obeso), definiendo como bajo peso, aquellos niños con un $\mathrm{Z}$ de IMC $<-1$, normal, entre $-1 \mathrm{y}+1$, sobrepeso entre $+1 \mathrm{y}+2$ y obeso $>+2$ (13-14). Se utilizó el test de Chi cuadrado para comparar los grupos control e intervenido con relación al tipo de alimentos que llevan de colación los niños. Se utilizaron las pruebas no paramétricas de signos de Wilcoxon (signed-rank test) para comparar la línea base con la intervención y la prueba de suma y rangos de Wilcoxon (Two-sample Wilcoxon rank-sum test) para comparar los grupos control e intervenido.

Todos los análisis estadísticos fueron realizados con STATA 10.1 para Windows (StataCorp. 2007. Stata Statistical Software: Release 10. College Station, TX: StataCorp LP.).

\section{RESULTADOS \\ Estado nutricional}

En la tabla 2 se aprecian los valores promedio del estado nutricional del total de la muestra. En el grupo intervenido se mantienen sin variación los valores de sobrepeso y obesidad. En el grupo control, el sobrepeso y obesidad presentan un leve aumento de $29 \%$ a $30.8 \%$ el sobrepeso, y de $21,1 \%$ a $21,5 \%$ la obesidad.

En la figura 1 se indica el porcentaje de obesidad por colegio. En el control se observa un leve aumento 
de la obesidad, mientras que en siete de los nueve colegios intervenidos se observó una disminución de la obesidad (entre un $4 \%$ a un $20 \%$ ). En un colegio intervenido, la obesidad tuvo un leve incremento y en otro, se produjo un fuerte incremento de la obesidad (sobre un 40\%). Al analizar los datos de este colegio por curso, se observó un aumento de la obesidad en todos los cursos evaluados.

En la figura 2 se indica el porcentaje de sobrepeso por colegio. En 6 de las escuelas intervenidas se encontró una disminución del sobrepeso, lo que es importante debido a que en estos mismos establecimientos también se produjo una disminución de la obesidad. Al existir un incremento importante del sobrepeso y la obesidad en

\section{TABLA 2}

Diagnóstico del estado nutricional de toda la muestra en la línea base y a los 6 meses de intervención.

\begin{tabular}{lccccc}
\hline \multicolumn{1}{|l}{ Intervención } & $\begin{array}{c}\text { Enflaquecidos } \\
\mathbf{n}(\boldsymbol{\%})\end{array}$ & $\begin{array}{c}\text { Normal } \\
\mathbf{n}(\boldsymbol{\%})\end{array}$ & $\begin{array}{c}\text { Sobrepeso } \\
\mathbf{n}(\boldsymbol{\%})\end{array}$ & $\begin{array}{c}\text { Obesidad } \\
\mathbf{n}(\boldsymbol{\%})\end{array}$ & $\begin{array}{c}\text { Total } \\
\text { Intervenidos } \\
\quad \text { Línea base }\end{array}$ \\
$\quad 32(2.6)$ & $606(49.6)$ & $345(28.2)$ & $239(19.6)$ & 1222 \\
$\quad$ Seguimiento 6 meses & $28(2.3)$ & $613(50.2)$ & $344(28.2)$ & $237(19.4)$ & 1222 \\
Control & $13(3.9)$ & $152(45.9)$ & $96(29)$ & $70(21.1)$ & 331 \\
$\quad$ Línea base & $12(3.6)$ & $146(44.1)$ & $102(30.8)$ & $71(21.5)$ & 331 \\
$\quad$ Seguimiento 6 meses & & & & & 1553 \\
Total & & & & &
\end{tabular}

\section{FIGURA 1}

Porcentaje de obesidad por colegio en el total de la muestra en la línea base y en el seguimiento.

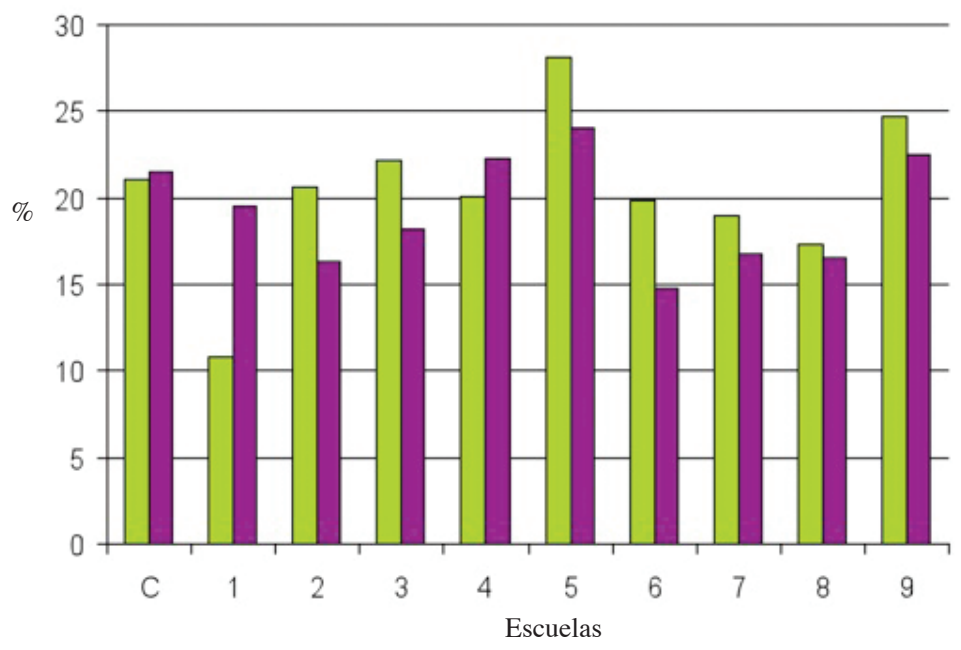


uno de los colegios intervenidos, se compensó la disminución en los otros colegios, resultando un total sin variación entre la línea de base y el seguimiento, como aparece en la tabla 2.

\section{FIGURA 2}

Porcentaje de sobrepeso por colegio en el total de la muestra en la línea base y en el seguimiento.

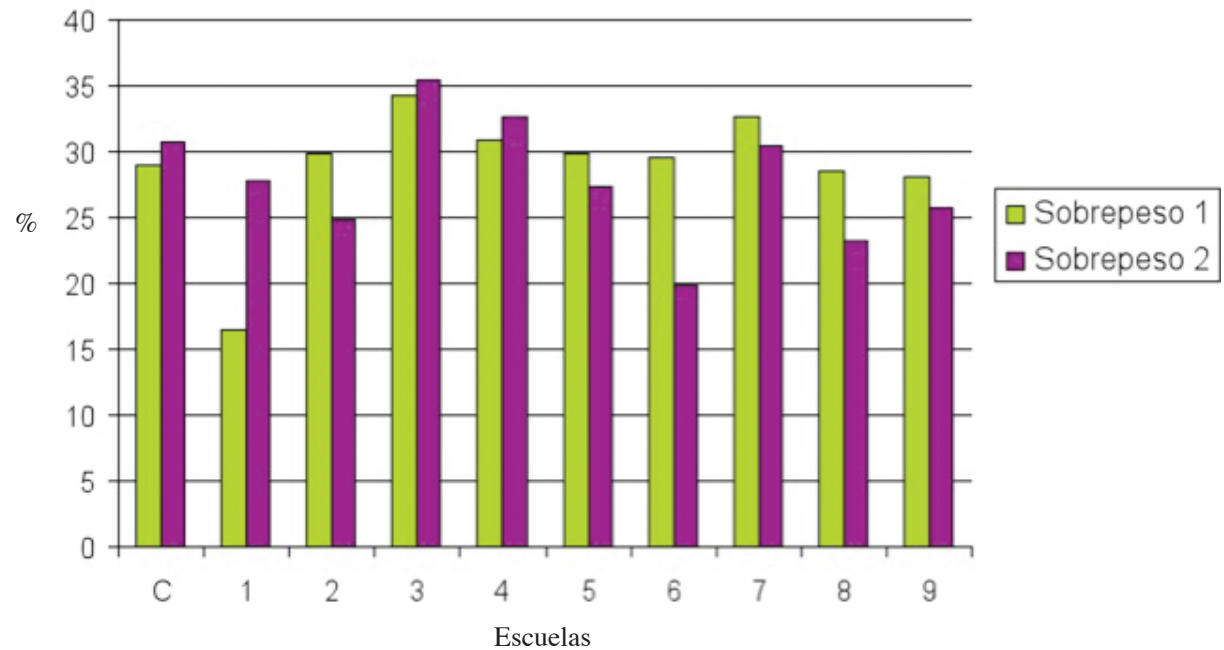

C: Escuela Control

1-9: Escuelas Intervenidas

Obesidad 1: Sobrepeso para la Línea base

Obesidad 2: Sobrepeso para el seguimiento a los 6 meses

\section{TABLA 3}

Tipo de alimentos que llevan de colación los niños de prekinder por género.

\begin{tabular}{|c|c|c|c|c|c|c|}
\hline \multirow[b]{2}{*}{ Alimentos } & \multicolumn{3}{|c|}{ Niños } & \multicolumn{3}{|c|}{ Niñas } \\
\hline & $\begin{array}{c}\text { Intervenidos } \\
\text { n (\%) } \\
102(46.6)\end{array}$ & $\begin{array}{c}\text { Control } \\
\text { n }(\%) \\
48(50.5)\end{array}$ & $\mathbf{p}$ & $\begin{array}{c}\text { Intervenidos } \\
\text { n }(\%) \\
117(53.4)\end{array}$ & $\begin{array}{l}\text { Control } \\
47 \text { (49.5) }\end{array}$ & $\begin{array}{c}\mathbf{p} \\
\mathbf{n}(\%)\end{array}$ \\
\hline Leche-yogurt & $18(17.7)$ & $10(20.8)$ & NS & 23 (19.7) & $12(25.5)$ & NS \\
\hline Jugo-bebidas & $36(35.3)$ & $21(43.8)$ & NS & $30(25.6)$ & $21(44.7)$ & $<0.05$ \\
\hline Galleta-queque & $15(14.7)$ & $10(20.8)$ & NS & $11(9.40)$ & $9(19.2)$ & NS \\
\hline $\mathrm{F} \& \mathrm{~V}$ & $16(15.7)$ & $3(6.3)$ & NS & $12(10.3)$ & $5(10.6)$ & NS \\
\hline Pan & $0(0)$ & $0(0)$ & & $1(6.4)$ & $3(2.4)$ & $<0.05$ \\
\hline Bocadillos dulces & $6(8.3)$ & $4(6.7)$ & NS & $6(5.1)$ & $1(2.1)$ & NS \\
\hline Bocadillos salados & $1(1.0)$ & $3(6.3)$ & NS & $3(12.8)$ & $6(5.5)$ & $<0.01$ \\
\hline Dinero & $3(2.9)$ & $3(6.3)$ & NS & $5(4.3)$ & $2(2.3)$ & NS \\
\hline
\end{tabular}




\section{Consumo y conocimiento de alimentos en los niños}

Consumo. En la tabla 3 se presentan los resultados de la evaluación del tipo de colaciones consumidas por los niños de prekinder, donde llama la atención el alto porcentaje que llevan jugos y bebidas como colación, siendo mayor en los niños y niñas del grupo control (43,8 \% y $44,7 \%$ respectivamente), con una diferencia significativa en las niñas. Más del $20 \%$ de niños y niñas lleva leche o yogur como colación.

La tabla 4 muestra los productos que llevan de colación los niños de kinder. En este caso continúa siendo alto el porcentaje de niños y niñas que llevan jugos y bebidas, destacándose su alto consumo en los varones del grupo intervenido $(51,9 \%)$.

En relación al consumo de alimentos en primero básico, la tabla 5 muestra que tanto el grupo control como el intervenido son iguales en la línea base, excepto para legumbres, en que el grupo control consume menos legumbres que el intervenido, siendo esta diferencia significativa, lo que no cambia después de la intervención. Se encontró un aumento significativo en el consumo de lácteos de 2,2 a 2,6 unidades diarias en el grupo intervenido. En segundo básico (tabla 6) no hay diferencias significativas en la línea base entre el grupo control y el intervenido, encontrándose diferencias significativas entre el inicio y final de la intervención en el consumo promedio de frutas, que aumentó de 2,5 a 3,2 frutas y en lácteos de 2 a 2,8 unidades diarias. En el grupo control, no se observaron diferencias en el consumo promedio de alimentos en ambos cursos, en los dos tiempos evaluados.

Conocimiento. Se analizaron las respuestas de la encuesta en relación a conocimiento en los niños de primero y segundo básico, apreciándose que no hubo diferencias entre el inicio y el final de la intervención en la mayoría, con excepción de tres de ellas. En la pregunta del alimento que puede reemplazar a la carne, el porcentaje de respuestas correctas disminuyó, respondiendo frutas en vez de legumbres por ser mejor conocido el efecto benéfico de las frutas que el de las legumbres. En la pregunta en la cual se debía destacar el efecto del pescado para mantener sano el corazón, la mayor parte respondió el choclo, lo que pudo deberse a que la intervención educativa del pescado se realizó al inicio de la intervención, sin llegar a profundizarse en el tema. En la pregunta que se refería a legumbres, el número de respuestas correctas fue bajo, lo que se debe al bajo conocimiento del valor nutricional que tienen las legumbres en la dieta de los chilenos.

\section{DISCUSIÓN}

La obesidad infantil ha continuado incrementándose en el país, a pesar de diferentes iniciativas tendientes a su reducción. Entre ellas, la de las EGO escuelas y el programa NutriRSE han sido insuficientes para enfrentar este grave problema. Por ello, es necesario continuar haciendo experiencias que puedan aportar elementos

\section{TABLA 4}

Tipo de alimentos que llevan de colación los niños de kínder por género.

\begin{tabular}{lcccccc} 
& \multicolumn{3}{c}{ Niños } & & & Niñas \\
Alimentos & $\begin{array}{c}\text { Intervenidos } \\
\mathbf{n}(\mathbf{\%}) \\
\mathbf{1 5 8}(\mathbf{5 2 . 7})\end{array}$ & $\begin{array}{c}\text { Control } \\
\mathbf{n}(\mathbf{\%})\end{array}$ & $\mathbf{p}$ & $\begin{array}{c}\text { Intervenidos } \\
\mathbf{4 0}(\mathbf{4 5 . 5})\end{array}$ & $\begin{array}{c}\text { Control } \\
\mathbf{n}(\mathbf{\%})\end{array}$ & $\mathbf{p}$ \\
& $22(13.9)$ & $8(20.0)$ & $\mathrm{NS}$ & $20(14.1)$ & $0(0)$ & $<0.01$ \\
\hline Leche-yogurt & $82(51.9)$ & $6(15.0)$ & $<0.0001$ & $54(29.2)$ & $14(35.8)$ & NS \\
Jugo-bebidas & $40(25.3)$ & $9(22.5)$ & NS & $42(29.6)$ & $9(18.8)$ & NS \\
Galleta-queque & $22(13.9)$ & $3(7.5)$ & NS & $15(10.6)$ & $1(2.1)$ & NS \\
F\&V & $1(0)$ & $0(0.5)$ & NS & $1(0.7)$ & $0(0)$ & NS \\
Pan & $13(8.2)$ & $3(7.5)$ & NS & $11(7.6)$ & $0(0)$ & $<0.05$ \\
Bocadillos dulces & $4(2.5)$ & $1(2.5)$ & NS & $7(4.9)$ & $1(2.1)$ & NS \\
Bocadillos salados & $13(8.2)$ & $1(2.5)$ & NS & $9(6.3)$ & $0(0)$ & NS \\
Dinero & & & & & &
\end{tabular}


positivos para establecer una política de Estado para prevenir la obesidad infantil, basada en educación en alimentación y nutrición y actividad física. Como es conocido, la enseñanza en alimentación y nutrición aún no se incorpora en el currículo de la educación preescolar y básica, por lo cual los resultados de este estudio pueden ser un aporte en ese sentido.

Lo interesante de la experiencia descrita en este

\section{TABLA 5}

Consumo promedio de algunos alimentos en el total de la muestra en la línea base y en el seguimiento en primero básico.

\section{Intervenidos $^{1}$}

\begin{tabular}{|c|c|c|c|c|c|c|}
\hline Alimentos & $\begin{array}{l}\text { Línea base } \\
\text { Promedio } \\
\text { (Min-Max) }\end{array}$ & $\begin{array}{c}\text { Seguimiento } 6 \text { meses } \\
\text { Promedio } \\
\text { (Min-Max) }\end{array}$ & $\mathbf{p}$ & $\begin{array}{l}\text { Línea base } \\
\text { Promedio } \\
\text { (Min-Max) }\end{array}$ & $\begin{array}{c}\text { Seguimiento } 6 \text { meses } \\
\text { Promedio } \\
\text { (Min-Max) }\end{array}$ & p \\
\hline $\begin{array}{l}\text { Verduras } \\
\text { (diario) }\end{array}$ & $\begin{array}{c}1.8 \\
(1-4)\end{array}$ & $\begin{array}{c}1.8 \\
(1-4)\end{array}$ & NS & $\begin{array}{c}1.7 \\
(1-4)\end{array}$ & $\begin{array}{c}1.9 \\
(1-4)\end{array}$ & NS \\
\hline $\begin{array}{l}\text { Frutas } \\
\text { (diario) }\end{array}$ & $\begin{array}{c}2.4 \\
(1-4)\end{array}$ & $\begin{array}{c}2.5 \\
(1-4)\end{array}$ & NS & $\begin{array}{c}2.2 \\
(1-4)\end{array}$ & $\begin{array}{c}2.7 \\
(2-4)\end{array}$ & NS \\
\hline $\begin{array}{l}\text { Lácteos } \\
\text { (diario) }\end{array}$ & $\begin{array}{c}2.2 \\
(1-4)\end{array}$ & $\begin{array}{c}2.6 \\
(1-4)\end{array}$ & $<0.05$ & $\begin{array}{c}2.1 \\
(1-4)\end{array}$ & $\begin{array}{c}2.5 \\
(1-4)\end{array}$ & NS \\
\hline $\begin{array}{l}\text { Legumbres } \\
\text { (semanal) }\end{array}$ & $\begin{array}{c}2.2 \\
(1-4)\end{array}$ & $\begin{array}{c}2 \\
(1-4)\end{array}$ & NS & $\begin{array}{c}1.6 \\
(1-4)\end{array}$ & $\begin{array}{c}1.6 \\
(1-4)\end{array}$ & NS \\
\hline
\end{tabular}

${ }^{1}$ Intervenidos=Control en línea base (NS) excepto legumbres $(\mathrm{p}<0.05)$

Min-Max: Mínimo-Máximo

\section{TABLA 6}

Consumo promedio de algunos alimentos en el total de la muestra en la línea base y en el seguimiento en segundo básico.

\section{Intervenidos ${ }^{1}$}

\begin{tabular}{|c|c|c|c|c|c|c|}
\hline Alimentos & $\begin{array}{l}\text { Línea base } \\
\text { Promedio } \\
\text { (Min-Max) }\end{array}$ & $\begin{array}{c}\text { Seguimiento } 6 \text { meses } \\
\text { Promedio } \\
\text { (Min-Max) }\end{array}$ & p & $\begin{array}{l}\text { Línea base } \\
\text { Promedio } \\
\text { (Min-Max) }\end{array}$ & $\begin{array}{c}\text { Seguimiento } 6 \text { meses } \\
\text { Promedio } \\
\text { (Min-Max) }\end{array}$ & p \\
\hline $\begin{array}{l}\text { Verduras } \\
\text { (diario) }\end{array}$ & $\begin{array}{c}2.1 \\
(1-4)\end{array}$ & $\begin{array}{c}2.2 \\
(1-4)\end{array}$ & NS & $\begin{array}{c}1.8 \\
(1-4)\end{array}$ & $\begin{array}{c}1.7 \\
(1-4)\end{array}$ & NS \\
\hline $\begin{array}{l}\text { Frutas } \\
\text { (diario) }\end{array}$ & $\begin{array}{c}2.5 \\
(1-4)\end{array}$ & $\begin{array}{c}3.2 \\
(1-4)\end{array}$ & $<0.001$ & $\begin{array}{c}2.8 \\
(1-4)\end{array}$ & $\begin{array}{c}2.7 \\
(1-4)\end{array}$ & NS \\
\hline $\begin{array}{l}\text { Lácteos } \\
\text { (diario) }\end{array}$ & $\begin{array}{c}2 \\
(1-4)\end{array}$ & $\begin{array}{c}2.8 \\
(1-4)\end{array}$ & $<0.0001$ & $\begin{array}{c}1.9 \\
(1-4)\end{array}$ & $\begin{array}{c}2.2 \\
(1-4)\end{array}$ & NS \\
\hline $\begin{array}{l}\text { Legumbres } \\
\text { (semanal) }\end{array}$ & $\begin{array}{c}2.2 \\
(1-4)\end{array}$ & $\begin{array}{c}2.2 \\
(1-4)\end{array}$ & NS & $\begin{array}{c}2.2 \\
(1-4)\end{array}$ & $\begin{array}{c}1.5 \\
(1-4)\end{array}$ & NS \\
\hline
\end{tabular}

${ }^{1}$ Intervenidos=Control en línea base (NS)

Min-Max: Mínimo-Máximo 
artículo es que una intervención en un año escolar bien estructurada, monitoreada y evaluada, se apreció una mejoría del estado nutricional en siete de los nueve colegios intervenidos, sin cambios en el colegio control. El otro aspecto interesante es que no existen diferencias en la intervención en actividad física entre los colegios intervenidos y el control, ya que existe un programa común para todo el municipio, establecido con antelación al inicio de este estudio. Por lo tanto, el elemento diferenciador lo constituye la educación en alimentación y nutrición. Para ello se realizó un trabajo intensivo con nutricionistas en el aula, que cada mes pusieron un tema nutricional para ser analizado en profundidad con los profesores y alumnos. Estos temas se pasaron en forma secuencial, por lo que difirieron en el tiempo en que fueron impartidas y la evaluación final.

Al analizar la obesidad en el total de los niños, ésta se mantuvo constante en los colegios intervenidos, aumentando levemente en el colegio control. Sin embargo, al analizar los datos de estado nutricional por colegio, la obesidad mejoró en siete de los nueve colegios intervenidos y en uno aumentó en forma importante. Esto último compensó la disminución en los otros seis colegios, resultando el promedio sin variación. La explicación de las diferencias entre las escuelas, puede estar dada por la por factores ambientales y por el compromiso de padres, apoderados y alumnos en el tema alimentarionutricional.

En cuanto al consumo de alimentos de los preescolares, llama la atención el alto porcentaje de niños que llevan jugos y bebidas como colación, lo cual significa un alto consumo de azúcar refinada, muy por encima del consumo de lácteos o frutas, que son alimentos saludables y muy recomendables. Esto coincide con lo encontrado en un estudio reciente realizado en la Región Metropolitana sobre preferencias de los niños en las colaciones (15). En los escolares de segundo básico, se observó un incremento significativo en el consumo de frutas y lácteos, entre el inicio y término de la intervención, lo que puede atribuirse a la educación realizada en estos dos alimentos. Esto se corrobora con las preguntas de conocimiento, donde hay una buena comprensión del tema frutas y lácteos, no así para pescado y legumbres.

Por tratarse sólo de un año escolar de seguimiento de los niños, aún no es posible sacar conclusiones definitivas. Sin embargo, este estudio permite indicar que a pesar del corto período de intervención en alimentación y nutrición, si ésta es bien realizada y evaluada, puede tener un impacto positivo en cambiar hábitos alimentarios y mejorar el estado nutricional de niños preescolares y del primer ciclo básico de la educación primaria.

\section{RESUMEN}

Se plantea una intervención en educación alimentaria y nutricional en nueve escuelas de la comuna de Peñalolén, con una escuela control. El objetivo es evaluar los cambios en el estado nutricional, conocimiento y consumo de alimentos de los niños de prebásica y básica durante el primer año de la intervención, comparados con el colegio control. Es un estudio cuasi-experimental, de seguimiento longitudinal en 1556 niños de prekinder, kínder, primero y segundo básico (1225 niños de las escuelas intervenidas y 331 de la escuela control) seguidos durante un año escolar. Se realizaron actividades educativas con profesores y alumnos y la evaluación consistió en una medición de su estado nutricional en marzo-abril y octubre-noviembre del año 2010; una medición de las colaciones en los niños de prekinder y kínder, con una encuesta alimentaria a los de primero y segundo básico. Los resultados mostraron que no hubo cambio en el estado nutricional en el total de los niños intervenidos, aumentando levemente el sobrepeso y obesidad en el colegio control. Sin embargo, al hacer el análisis por colegio, en siete de los nueve colegios intervenidos disminuyó la obesidad. En cuanto a consumo, un alto porcentaje de niños de prebásica llevaban jugos y bebidas en las colaciones y en los niños de básica se produjo un incremento significativo del consumo de frutas y lácteos durante el año. En conocimiento, los niños de primero y segundo básico tuvieron una buena comprensión en frutas y lácteos, no así para el pescado y legumbres. A pesar del corto período de intervención, este estudio permite señalar que cuando las intervenciones en alimentación y nutrición se realizan en forma estructurada y son evaluadas, pueden tener un impacto positivo en el cambio de hábitos alimentarios y en la disminución de la obesidad en preescolares y escolares de escuelas municipales.

Palabras clave: evaluación estado nutricional, obesidad pre escolar y escolar, conocimiento alimentario, consumo alimentario.

Dirigir la correspondencia a:

Dr. Fernando Vio

INTA, Universidad de Chile

El Líbano 5524, Macul

Santiago, Chile

Fono 56-2-9781576

E-mail: fvio@inta.uchile.cl

Agradecimientos: Este estudio fue financiado por la Empresa Nestlé Chile en colaboración con la Corporación Municipal de Peñalolén. 
La empresa que financió el estudio no tuvo influencia en el diseño, ni en el análisis ni lugar de publicación de los resultados.

\section{BIBLIOGRAFÍA}

1. Vio F, Albala C, Kain J. Nutrition transition in Chile revisited: mid-term evaluation of obesity goals for the period 2000-2010. Public Health Nutr 2008; 11:405-12.

2. WNAEB Www junaeh cl/prontus junaeh/site/ artic/20100625/pags/20100625095833.htm. Accedido en abril 2011.

3. Kain J, Leyton B, Cerda R, Vio F, Uauy R. Twoyear controlled effectiveness trial of a school-based intervention to prevent obesity in Chilean children. Public Health Nutr 2009; 12:1451-61.

4. Kain J, Uauy R, Leyton B, Cerda R, Olivares S, Vio F. Efectividad de una intervención en educación alimentaria y actividad física para prevenir obesidad en escolares de la ciudad de Casablanca, Chile (2003-2004) Rev Med Chil 2008; 136:22-30.

5. Kain J, Vio F, Leyton B, Cerda R, Olivares S, Uauy R, Albala C. Estrategia de promoción de la salud en escolares de Educación Básica municipalizada de la comuna de Casablanca, Chile. Rev Chil Nutr 2005; 32:126-132.

6. Gobierno de Chile. Ministerio de Salud. Estrategia Global_contra la Ohesidad (EGO_Chile) http:// www.minsal.cl/ici/nutricion/nutricion .html. Accedido en abril 2011.

7. NutriRSE. Recomendaciones para gestionar programas de vida saludable. Abril 2011. Acción RSE, Santiago, Chile.
8. Facultad de Medicina. Universidad de Chile. Tercer Informe. Evaluación externa de las acciones realizadas en las EGO-escuelas para el Ministerio de Salud. Santiago, enero 2010.

9. Ministerio de Salud/INTA U. de Chile/VIDA CHILE. Guías para una Vida Saludable. Santiago, Ministerio de Salud. Norma General Técnica No 76 , 10 de agosto 2005.

10. Bustos N, Benavides C.ii Qué rico es comer sano!! Programación de actividades para el fomento de las Guías Alimentarias Chilenas en el primer ciclo de la enseñanza básica. Santiago, Universidad de Chile;2009

11. Ministerio de Agricultura; Universidad de Chile, INTA; Corporación 5 al Día. Contribución de la Política Agraria al Consumo de Frutas y Verduras en Chile. Un Compromiso con la Nutrición y la Salud en la Población. Santiago: Maval Ltda. Santiago, 2008.

12. World Health Organization (WHO). Physical status: The use and interpretation of Anthropometry. Report a WHO Expert Committee Technical Report Series, No 854, Geneva, 1995

13. World Health Organizaton (WHO). The WHO Child Growth Standards 2006: http://www.who. int/childgrowth/standards/en/ Geneva, Switzerland.

14. World Health Organization (WHO). The WHO 2007 SAS Macro Package. World Health Organization (WHO). Geneva, Switzerland. 2007.

15. Bustos N, Kain J, Leyton B, Olivares S, Vio F. Colaciones habitualmente consumidas por niños de escuelas municipalizadas: Motivaciones para su elección. Rev Chil Nutr 2010; 37:178-83. 